\title{
Treatment of Neurodegenerative Ataxias With Intravenous Immune Globulin
}

\author{
Theresa A. Zesiewicz ${ }^{\mathrm{a}, \mathrm{d}}$, Seok Hun Kim ${ }^{\mathrm{b}}$, Kelly L. Sullivan ${ }^{\mathrm{a}}$, Jeannie Stephenson ${ }^{\mathrm{b}}$, \\ Israt Jahan a , Susan L. Perlman ${ }^{c}$, Jessica D. Shaw ${ }^{a}$, Tuan Vu ${ }^{a}$, Clifton Gooch ${ }^{\mathrm{a}}$
}

\begin{abstract}
Background: Neurodegenerative ataxias, including spinocerebellar ataxias (SCAs), are progressive diseases without effective treatment. There is preclinical evidence that inflammation may contribute to neuronal injury in several neurodegenerative ataxias. Intravenous immune globulin (IVIG) is a therapeutic modality that is used as treatment of several autoimmune and inflammatory disorders.
\end{abstract}

Methods: The primary objective of this open-label pilot study was to assess the effect of IVIG on neurodegenerative ataxias as measured by total scale for the assessment and rating of ataxia (SARA) score. Three patients received IVIG $(2 \mathrm{~g} / \mathrm{kg}$ of body weight, divided over 5 days) once monthly for 3 months, and were evaluated before the first infusion, 2 weeks after each infusion, as well as 28 and 56 days following the final course of treatment. Secondary measures included SARA subsection scores and gait assessment using the GAITRite ${ }^{\circledR}$ Walkway System. Another SCA 3 patient at a different site was treated with six monthly courses of IVIG, and assessed with the SARA score.

Results: Three out of five patients completed the open-label study (SCA 3, a neurodegenerative ataxia associated with an aprataxin genetic variant, and late onset cerebellar ataxia (LOCA) patient). All three patients demonstrated improvement in the SARA score following IVIG. SARA total scores improved by $30-50 \%$ and gait sub-scores improved by $20-50 \%$ after the last course of IVIG. Clinical improvements attenuated approximately 2 months following

Manuscript accepted for publication August 27, 2014

${ }^{a}$ Department of Neurology, University of South Florida Morsani College of Medicine, Tampa, FL, USA

${ }^{\mathrm{b}}$ School of Physical Therapy \& Rehabilitation Sciences, University of South Florida Morsani College of Medicine, Tampa, FL, USA

${ }^{\mathrm{c}}$ Department of Neurology, University of California, Los Angeles

(UCLA), Los Angeles, CA, USA

${ }^{\mathrm{d}}$ Corresponding Author: Theresa A. Zesiewicz, Department of Neurology, University of South Florida, Parkinson's Disease and Movement Disorders Center, 12901 Bruce B. Downs Blvd., MDC 55, Tampa, FL 33612, USA. Email: tzesiewi@health.usf.edu

doi: http://dx.doi.org/10.14740/jnr291w the last infusion. The SCA 3 patient treated at another site with a longer protocol had even greater improvement in the SARA score.

Conclusions: IVIG may have therapeutic efficacy in neurodegenerative ataxias, including SCA and LOCA.

Keywords: Ataxia; Intravenous immune globulin; Spinocerebellar ataxia

\section{Introduction}

Cerebellar disease causes substantial impairment in affected subjects, including imbalance, a wide-based, ataxic gait, incoordination, and speech and swallowing disorders, among other symptoms. The cerebellum can be damaged by metabolic disturbances, infections, ischemia, inflammatory disease, neoplasms, and neurodegenerative diseases, particularly the heterofamilial ataxias, including spinocerebellar ataxias (SCAs) and multiple-system atrophy (MSA). The SCAs are autosomal dominant neurodegenerative disorders that are frequently caused by an expanded triplicate (CAG) repeat in the coding region of the ataxin protein. There are currently no effective treatments for neurodegenerative ataxias, although drugs such as riluzole [1] and varenicline [2-4] have shown some therapeutic promise.

Inflammation is a well-known cause of central nervous system and cerebellar injury, and may also contribute to neuronal injury in the neurodegenerative ataxias [5]. Intravenous immune globulin (IVIG) is an immunomodulator that suppresses inflammation, and is an effective treatment for many autoimmune and inflammatory disorders. Here we report the results of IVIG administration to three patients with differing SCAs.

\section{Materials and Methods}

The primary objective of the pilot study performed at the University of South Florida (USF, Tampa, FL, USA) was to assess the effect of IVIG on neurodegenerative ataxia 


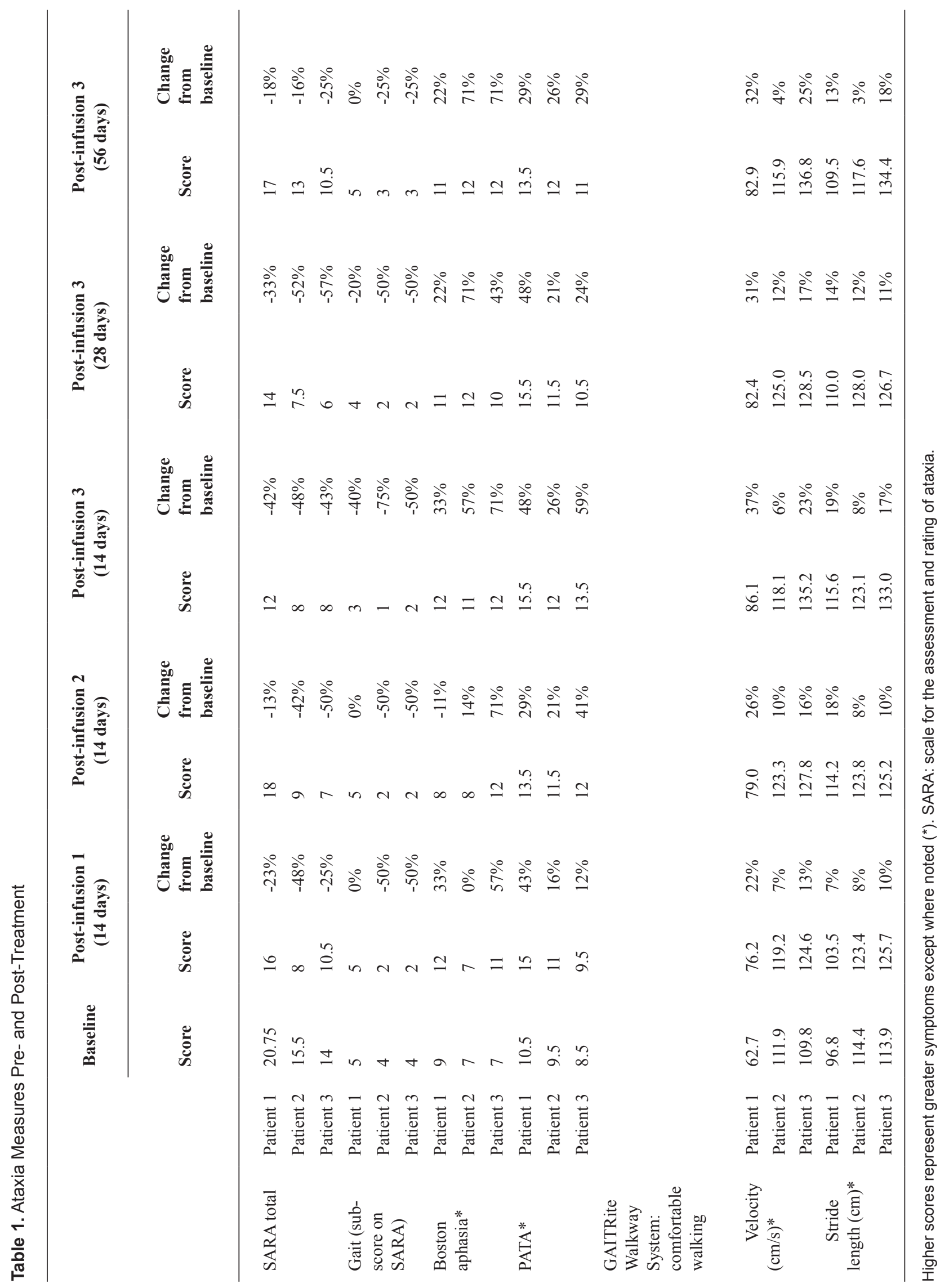


as measured by total scale for the assessment and rating of ataxia (SARA) score [6]. Patients of 10 - 80 years old, with neurodegenerative ataxia diagnosed by a movement disorders expert (TAZ), the ability to ambulate 30 feet (with or without an assistive device), and stable medications for 30 days prior to baseline were considered for inclusion. Patients with any other potential cause of cerebellar dysfunction, including vitamin deficiencies, diabetes, vasculitis, other concurrent neurological disease were excluded, as were patients with IgA deficiency or any contraindication to IVIG therapy.

All patients received courses of IVIG $(2 \mathrm{~g} / \mathrm{kg}$ of body weight divided over 5 days) during weeks 1, 5, and 9, for a total of three full courses of treatment. Patients were evaluated prior to the first course, 14 days after each course, and 28 days and 56 days after the final course. IVIG was supplied by Baxter ${ }^{\circledR}$ Pharmaceuticals, United States. Secondary measures included SARA scores, PATA test [7], the oral motor function subsection of the Boston Diagnostic Aphasia Examination [8, 9], and gait assessment using the GAITRite ${ }^{\circledR}$ Walkway System (CIR Systems, Inc., Sparta, NJ, USA). For gait assessment, patients completed five trials of walking on a 26-foot walkway at their comfortable walking speed across the testing sessions. Gait velocity and stride length were calculated to assess changes in walking ability following IVIG treatments. The study was approved by the USF IRB and was listed on clinicaltrials.gov as NCT01350440.

\section{Results}

Five patients were initially enrolled in the study, with three patients completing. One patient withdrew due to an adverse event (eye irritation) and one screened patient was diagnosed with hereditary spastic paraparesis and removed from the study. Neither of these patients was included in the analysis. The three remaining patients completing the study included one subject with SCA 3 (69/21 CAG repeats), one subject with neurodegenerative ataxia associated with a DNA sequence variant in the aprataxin gene (APTX variant 1 - transition $\mathrm{A}>\mathrm{G}$; nucleotide position 183; codon position 61 ) and one subject with idiopathic late onset cerebellar ataxia (LOCA), based on clinical observations [10]. Genetic tests were performed by Athena Diagnostics. The mean age of the patients was 66.7 years, and the mean disease duration was 21.3 years. The SCA 3 subject had been previously treated with varenicline, off-label for a period of 6 years. Neurological examinations demonstrated cerebellar dysfunction in all patients. Sensory deficits, including distal, symmetric stocking distribution reduction to all modalities, were noted in the SCA 3 and LOCA patients and confirmed by nerve conduction studies which showed distal symmetric axonal sensory polyneuropathy (Table 1 for baseline SARA data).

Following IVIG treatment, all three patients demonstrated improvement in the SARA score (Table 1). Twenty-eight days after the third course of IVIG, SARA total scores improved $30-50 \%$, with notable improvements in the gait subscore (20-50\% improvement). Other measures of cerebellar dysfunction, including speech and oral motor function as measured by the Boston Diagnostic Aphasia Examination, improved from $22 \%$ to $71 \%$ following the third infusion. Clinical improvements were maintained for 28 days after the final infusion, but attenuated by day 56. Patients also showed noticeably better objective measures of comfortable walking using the GAITRite ${ }^{\circledR}$ Walkway System. Gait velocity and stride length increased in all patients (range 12-31\% and 11$14 \%$, respectively) 28 days after the third course of IVIG.

The effect of IVIG was also evaluated in a 49-year-old woman with SCA 3 who was treated at the University of California, Los Angeles (UCLA) by SLP. The patient was of Asian ancestry, with genetically confirmed SCA 3 (repeats of 72/14). Her symptoms included diplopia, syncope, muscle cramping, paresthesias, and imbalance. She had previously received off-label treatment for cerebellar dysfunction with amantadine, meclizine, riluzole, and 4-aminopyridine, as well as physical therapy and vestibular rehabilitation, without benefit. Her neurological exam included abnormalities in saccadic pursuit, mild bilateral limb dysmetria, mild gait and stance dysfunction. The patient was treated with IVIG $2 \mathrm{~g} / \mathrm{kg}$ monthly starting in October 2013. Following two courses of IVIG, her SARA total score decreased from 4.5 to 1 ; following four courses of IVIG, the SARA score was 2.5; following six rounds of IVIG, her SARA score was 0. Symptomatically, the patient reported improvements in dizziness, depth perception, cramps and stiffness, although parethesias of her feet were unchanged.

\section{Discussion}

Inflammation is known to worsen injury in tissues affected by disease or trauma, and therapeutic regimens often incorporate anti-inflammatory treatments. Inflammation may also contribute to neuronal injury in neurodegenerative ataxias [5]. The role of inflammation as a co-conspirator in the propagation of neuronal injury and death has been well documented in neurodegenerative diseases [11]. IVIG is a therapeutic modality that is used as treatment of several autoimmune and inflammatory disorders. In one immunohistochemical study, human SCA 3 pontine neurons strongly expressed the anti-inflammatory interleukin-1 receptor antagonist, the pro-inflammatory cytokine interleukin-1beta, and the pro-inflammatory chemokine SDF1 [5]. Increased numbers of reactive astrocytes and activated microglial cells were also identified in SCA 3 pontine regions. Another openlabel pilot study performed in nine patients with the neurodegenerative disorder MSA successfully used monthly infusions of the IVIG preparation Privigen ${ }^{\circledR}$ (dose $0.4 \mathrm{~g} / \mathrm{kg}$ for 6 months) to effect significant improvements in the Unified 
Multiple System Atrophy Rating Scale (UMSARS)-I (activities of daily living) and UMSARS-II (motor functions) at endpoint relative to baseline [12].

In this report, IVIG reduced ataxia symptoms in three patients with SCA, as demonstrated by both clinical rating scales and by quantitative temporospatial gait analysis, with maximal improvement paralleling projected maximal IVIG effect. Later attenuation in improvement paralleled expected pharmacokinetic declines in IVIG levels. Although the small sample size and lack of placebo controls limit the generalizability of these results, further controlled studies of IVIG in the treatment of neurodegenerative ataxias, including SCA, are warranted.

\section{Financial Disclosures}

Theresa A. Zesiewicz, MD FAAN has received consulting fees from TEVA, GE Healthcare and UCB Pharma, research grant support Astellas Pharmaceuticals, Friedreich's Ataxia Research Alliance, Edison Pharmaceuticals, Takeda, GlaxoSmithKline, and Baxter Pharmaceuticals. Seok Hun Kim, PT, PhD reports no disclosures. Kelly L. Sullivan PhD reports no disclosures. Jeannie Stephenson, PT, MS, NCS reports no disclosures. Israt Jahan MD reports no disclosures. Jessica D. Shaw, MPH reports no disclosures. Susan Perlman, MD, has received research grant support from the Friedreich's Ataxia Research Alliance, Edison Pharmaceuticals, and ViroPharma/Shire. Tuan Vu MD reports no disclosures. Clifton Gooch, MD FAAN is a research consultant for NeuralStem, Baxter and CSL Behring, a medical advisory board member of the GBS/CIDP Foundation International, and an employee of the FDA. He receives travel funding from the NIH as Chair of an NIH Data Safety Monitoring Board.

\section{Grant Support}

Friedreich's Ataxia Research Alliance (FARA), IVIG supplied by Baxter Pharmaceuticals.

\section{References}

1. Ristori G, Romano S, Visconti A, Cannoni S, Spadaro
M, Frontali M, Pontieri FE, et al. Riluzole in cerebellar ataxia: a randomized, double-blind, placebo-controlled pilot trial. Neurology. 2010;74(10):839-845.

2. Zesiewicz TA, Sullivan KL. Treatment of ataxia and imbalance with varenicline (chantix): report of 2 patients with spinocerebellar ataxia (types 3 and 14). Clin Neuropharmacol. 2008;31(6):363-365.

3. Zesiewicz TA, Sullivan KL, Gooch CL, Lynch DR. Subjective improvement in proprioception in 2 patients with atypical Friedreich ataxia treated with varenicline (Chantix). J Clin Neuromuscul Dis. 2009;10(4):191193.

4. Zesiewicz TA, Sullivan KL, Freeman A, Juncos JL. Treatment of imbalance with varenicline Chantix(R): report of a patient with fragile $\mathrm{X}$ tremor/ataxia syndrome. Acta Neurol Scand. 2009;119(2):135-138.

5. Evert BO, Vogt IR, Kindermann C, Ozimek L, de Vos RA, Brunt ER, Schmitt I, et al. Inflammatory genes are upregulated in expanded ataxin-3-expressing cell lines and spinocerebellar ataxia type 3 brains. J Neurosci. 2001;21(15):5389-5396.

6. Schmitz-Hubsch T, du Montcel ST, Baliko L, Berciano J, Boesch S, Depondt C, Giunti P, et al. Scale for the assessment and rating of ataxia: development of a new clinical scale. Neurology. 2006;66(11):1717-1720.

7. Subramony SH, May W, Lynch D, Gomez C, Fischbeck K, Hallett M, Taylor P, et al. Measuring Friedreich ataxia: Interrater reliability of a neurologic rating scale. Neurology. 2005;64(7):1261-1262.

8. Naeser MA, Hayward RW. Lesion localization in aphasia with cranial computed tomography and the Boston Diagnostic Aphasia Exam. Neurology. 1978;28(6):545551 .

9. Spreen O, Risser AH. Assessment of aphasia. Oxford University Press. 2003. ISBN 0-19-514075-3.

10. Harding AE. The clinical features and classification of the late onset autosomal dominant cerebellar ataxias. A study of 11 families, including descendants of the 'the Drew family of Walworth'. Brain. 1982;105(Pt 1):128.

11. Amor S, Puentes F, Baker D, van der Valk P. Inflammation in neurodegenerative diseases. Immunology. 2010;129(2):154-169.

12. Novak P, Williams A, Ravin P, Zurkiya O, Abduljalil A, Novak V. Treatment of multiple system atrophy using intravenous immunoglobulin. BMC Neurol. 2012;12:131. 